\title{
LOS DINOSAURIOS DE MORELLA (CASTELLÓN, ESPAÑA): HISTORIA DE SU INVESTIGACION
}

\author{
José Miguel GASULLA ASENSIO \\ Unidad de Paleontología. Departamento de Biología. Facultad de Ciencias. Uni- \\ versidad Autónoma. Cantoblanco, 28049 - Madrid. E-mail: gasuviol@terra.es
}

Gasulla Asensio, J. M. 2005. Los dinosaurios de Morella (Castellón, España): historia de su investigacion. [The dinosaurs of Morella (Castellón, Spain): History of their investigation.] Revista Española de Paleontología, N.E. X, 29-38. ISSN 0213-6937.

\begin{abstract}
The fossiliferous site of Morella has always been linked to the history of the Spanish dinosaurology. The first bony evidences are known during the late XIX century, by Nicolás Ferrer y Julve and Juan Vilanova y Piera, later José Royo y Gómez published specific studies and later to the publication of the first Spanish monograph on the Iberian Peninsula dinosaurs. The investigation on dinosaurs and their popularization have increased notably in Morella during the last ten years associated to the realization of the Museum "Temps de dinosaures", to new recent discoveries at that time in ichnofossils in Vallivana, and to a permanent paleontological vigilance of open air fossiliferous clay quarry in Mas de la Parreta.
\end{abstract}

Keywords: Lower Aptian, Arcillas de Morella Formation, history of the research, dinosaurs.

\section{RESUMEN}

Desde que, en el último tercio del siglo XIX, se describen las primeras evidencias directas de dinosaurios en España, los yacimientos de Morella siempre han estado ligados a la historia de la dinosauriología española. Primero con la investigación de Nicolás Ferrer y Julve y Juan Vilanova y Piera, luego con el estudio más específico de José Royo y Gómez y más tarde con la publicación de la primera monografía sobre dinosaurios de la península Ibérica. En los últimos diez años, la investigación y divulgación en dinosaurios se ha incrementado notablemente en Morella, entre otras cosas, por la realización del museo monotemático "Temps de dinosaures", por la localización del yacimiento de icnitas de Vallivana o por el control paleontológico permanente de una cantera de arcillas en el Mas de la Parreta.

Palabras clave: Aptiense inferior, Formación Arcillas de Morella, historia de la investigación, dinosaurios.

\section{INTRODUCCIÓN}

El desarrollo histórico del estudio de los dinosaurios en España está estrechamente ligado a la evidencia aportada por los yacimientos de Morella. La investigación realizada sobre fósiles descubiertos en las localidades morellanas ha sido fundamental para el desarrollo de dicho estudio y pionera en esta disciplina. Hay que tener presente que, el conocimiento de los yacimientos pertenecientes a la Formación Arcillas de Morella (Aptiense inferior) se remonta al último tercio del siglo XIX, coincidiendo con los primeros pasos de la dinosauriología española.

Estos primeros pasos, realizados principalmente por Juan Vilanova y Piera fueron seguidos, en el primer tercio del si- glo XX, por José Royo y Gómez con sus estudios específicos sobre los dinosaurios españoles, principalmente de hallazgos realizados en las localidades morellanas. Royo y Gómez fue el primer investigador que abordó las listas faunísticas de algunos yacimientos de dinosaurios en España y aportó una importante colección de restos a las colecciones del Museo Nacional de Ciencias Naturales de Madrid.

Aunque se realizan algunos trabajos en los años cincuenta y sesenta, sobre todo por el investigador francés Albert de Lapparent, se produce un lapso en la historia de la dinosauriología española y, de nuevo, Morella está implicada en buena parte de su resurgimiento. Alrededor de cincuenta años después de los trabajos de Royo y Gómez, a principios de la década de los ochenta, los inves- 
tigadores José V. Santafé, Ma Lourdes Casanovas, José Luis Sanz y Sebastián Calzada publican la primera monografía sobre dinosaurios de España, precisamente con materiales procedentes de yacimientos morellanos, tanto de excavaciones sistemáticas como otros aportados por aficionados locales.

La investigación y divulgación de los dinosaurios de Morella se ha incrementado notablemente en los últimos diez años, probablemente como consecuencia de la evidente revalorización de la que se están beneficiando los recursos paleontológicos a todos los niveles. Dicha revalorización propició la creación del Museo "Temps de dinosaures" en 1994, uno de los primeros museos específicos en dinosaurios de España. Además, supuso un importante apoyo a los aficionados o estudiosos interesados por los fósiles de la localidad. En 1997 se descubrieron las primeras evidencias indirectas de dinosaurios con el yacimiento de icnitas de terópodos de Vallivana, permitiendo que Morella se incorporase al importante conjunto de yacimientos con huellas de dinosaurios en España. La investigación actual de Morella también se ha visto beneficiada, por una parte, con el desarrollo de normativas de gestión de los recursos paleontológicos, y por otra, con la sensibilidad en la protección de los bienes culturales de la empresa que explota la cantera de arcillas establecida en el Mas de la Parreta de Morella. Los controles paleontológicos realizados desde el año 2000 han incrementado notablemente la información sobre la geología y paleontología de la Formación Arcillas de Morella, tanto por la aportación de una ingente cantidad de restos fósiles, como por la incorporación al trabajo de investigación de un equipo multidisciplinar compuesto por especialistas en diferentes campos de ambas disciplinas.

\section{LOS PRIMEROS DESCUBRIMIENTOS EN MORELLA}

Los yacimientos con evidencias directas de restos de dinosaurios de Morella se conocen, como se ha dicho, desde la segunda mitad del siglo XIX. Los primeros indicios escritos sobre descubrimientos de restos fósiles, sin duda relacionados con dinosaurios, fueron narrados por el historiador morellano, José Segura y Barreda (1815-1888). En su libro "Morella y sus aldeas", en el apartado de la Epoca Antigua, se muestra crítico con algunos autores coetáneos respecto a la presencia del hombre en España antes del diluvio universal y matiza:

\footnotetext{
"Nosotros hemos tenido en nuestras manos algunos huesos disformes, encontrados alrededor de Morella en estado de fosilización, no pueden ser de alguna especie conocida en nuestros tiempos, pero no diremos que son de jigante, por que sabido es, que el Diluvio hizo desaparecer animales, ahora desconocidos " (Segura y Barreda, 1868, T. II: 280).
}

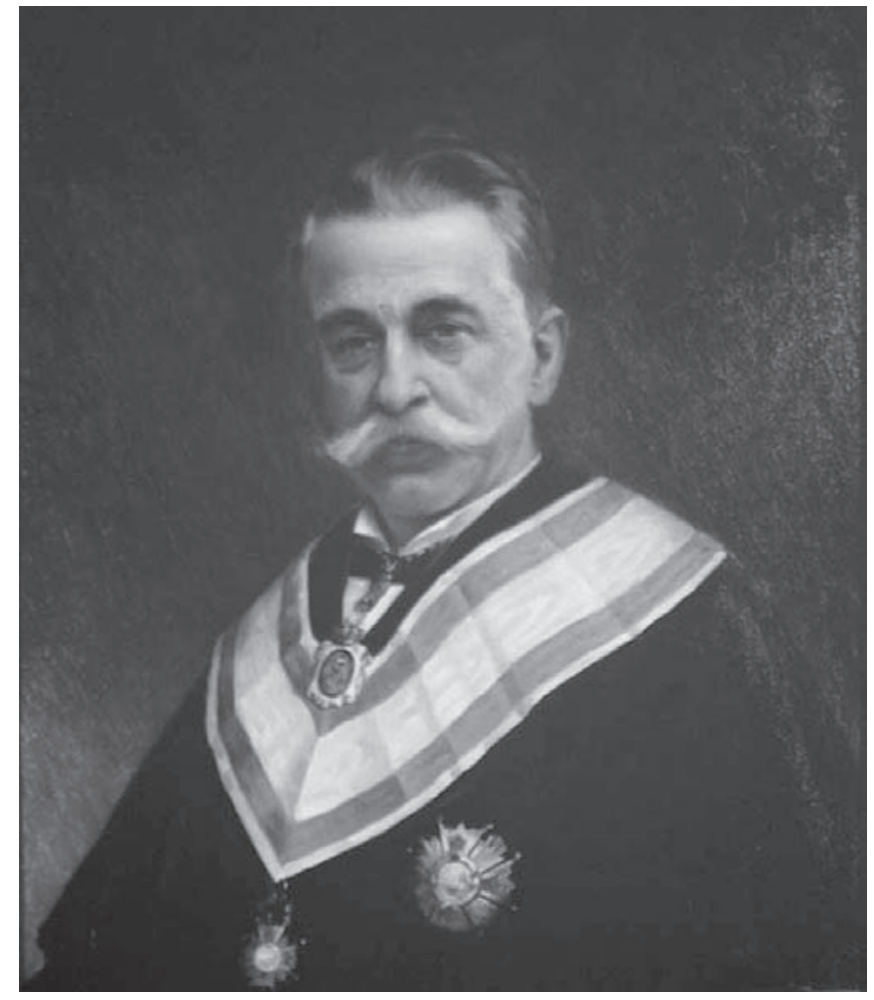

Figura 1. Nicolás Ferrer y Julve (1839-1901). Archivo Universitat de València

A esta aseveración, adjunta una nota a pie de página indicando que el hallazgo había sido realizado por Nicolás Ferrer y Julve (1839-1901), en agosto de 1868 (Fig. 1). Quien con posterioridad fue catedrático de Anatomía quirúrgica y Rector de la Universidad de Valencia. Ferrer y Julve, originario de la población turolense de Mirambel, cercana a Morella y a su comarca (Els Ports), realizó numerosas investigaciones arqueológicas y paleontológicas en esta zona. Segura y Barreda afirma que éste encontró:

... algunos huesos disformes en las cercanías de Morella, que conservan no solo la figura esterior, sino hasta la médula fosilizada." (Segura y Barreda, 1868, T. II: 280).

Otro historiador local, Francisco Ortí (1958) afirma que Ferrer y Julve halló restos de un esqueleto de dinosaurio. Evidentemente, en ese momento, tanto Segura y Barreda como Ferrer y Julve carecían de los conocimientos para saber de que se trataba. Sin embargo, parece que, por el tamaño, por el comentado estado de fosilización e incluso por la situación espacial de su hallazgo, se trataría de restos de dinosaurios. Posteriormente, quien así lo confirmaría, sería el investigador valenciano Juan Vilanova y Piera (1821-1883) (Fig. 2), primer catedrático de Geología y Paleontología de la Universidad Central de Madrid desde 1852 hasta 1878. En esta última fecha, se disgregaron las dos disciplinas, quedándose Vilanova con la cátedra de Paleontología (Gozalo \& Salavert, 1995). 


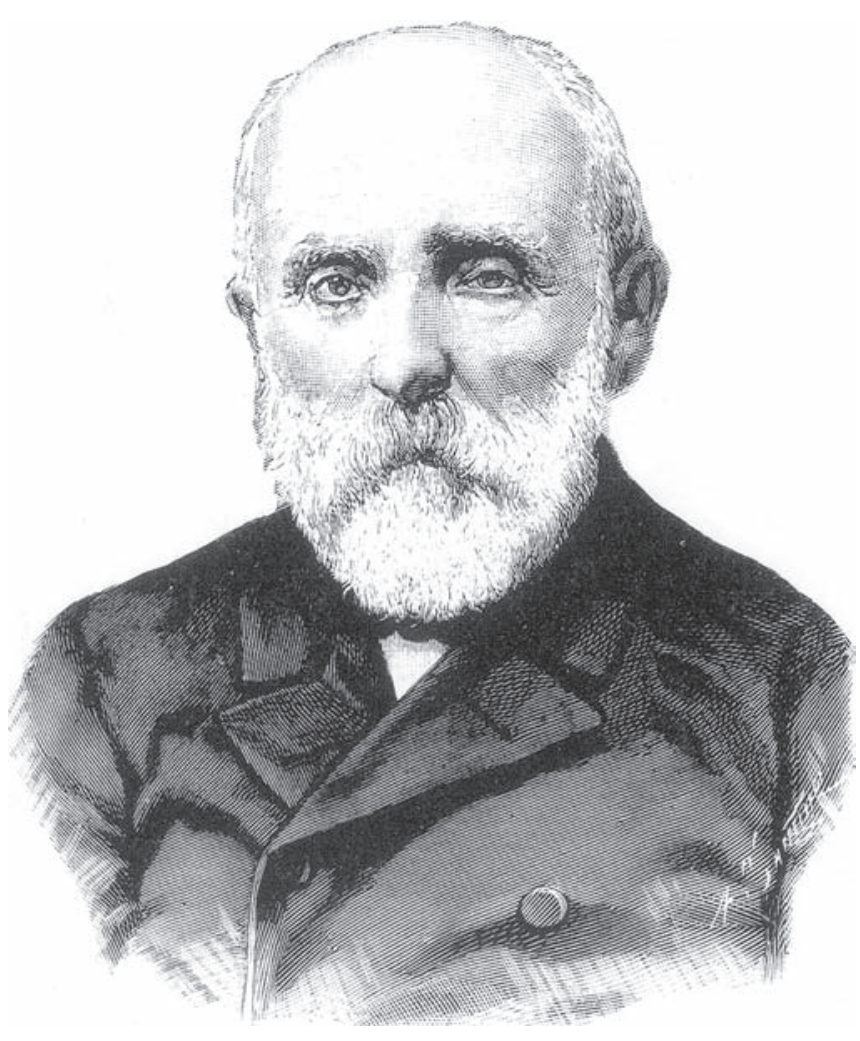

Figura 2. Juan Vilanova y Piera (1821-1883).

Vilanova y Piera realizó la primera cita sobre restos de dinosaurios de España en su libro "Compendio de Geología” publicado el año 1872, en él indica:

"Para concluir, debo mencionar el hallazgo hecho tres años há por D. Nicolás Ferrer, y confirmado más tarde por mí, de varios restos de un reptil colosal al pié de la colina llamada Benigania, junto á los muros mismos de Morella. Tambien poseo dos huesos largos, que probablemente pertenecen al Ignanodon [sic] Mantelli que, procedentes de Utrillas, me mandó hace poco el distinguido médico de Montalban D. Jerónimo Balduque. Quizás sean estos los únicos huesos de reptiles cretáceos hasta el presente encontrados en la Península, razon que me ha movido á dar estas noticias." (Vilanova y Piera, 1872: 477).

Para corroborar estas noticias, Vilanova y Piera (1873) comunicó a la Real Sociedad Española de Historia Natural que aquellos restos fósiles localizados en Morella y Utrillas estaban en su poder y que además podrían pertenecer a Iguanodon.

Posteriormente Ferrer y Julve (1877) redacta un documento de donación de una colección de fósiles recogidos en Morella. Entre ellos, destaca un probable hueso de dinosaurio que regaló al Museo de Historia Natural de la Universidad de Valencia:

"Mas debe hacer presente el espositor, que por el tamaño y el peso, tiene el sentimiento de no poder presentar un femur colosal, gigantesco, encontrado por él mismo en la Benigania, inmediaciones de Morella, a 800 metros sobre el nivel del mar, en medio de bancos de arcillas irisadas y debajo de capas cuarteadas de caliza, restos tal vez de algún gran saurio antediluviano, por cuyo hueso, perfectamente conservado y regalado ál Museo de Historia natural de la Universidad de Valencia, podria deducirse la organizacion del animal á que perteneció." (Ferrer y Julve, 1877).

Este hueso, junto con todos los ejemplares de las colecciones de mineralogía y paleontología del Museo de Historia Natural, desaparecieron durante el incendio que, en 1932 asoló un ala de la Facultad de Ciencias de la Universidad Literaria de Valencia (García, 1999).

\section{JOSÉ ROYO Y GÓMEZ}

Aunque estos fueron los primeros indicios en el estudio de dinosaurios, sin duda, José Royo y Gómez (1895-1961; Fig. 3) fue el primer paleontólogo español que los estudió específicamente (Sanz, 1996). La investigación en dinosaurios de este científico y político castellonense se fundamentó básicamente en los materiales fósiles localizados en los yacimientos morellanos (Alcalá \& Alcalá, 1996). Su investigación en Morella contó además con la colaboración de otros importantes estudiosos castellonenses como fueron Francisco Beltrán Bigorra (1886-1962), catedrático de Biología de la Facultad de Ciencias de Valencia y Vi-

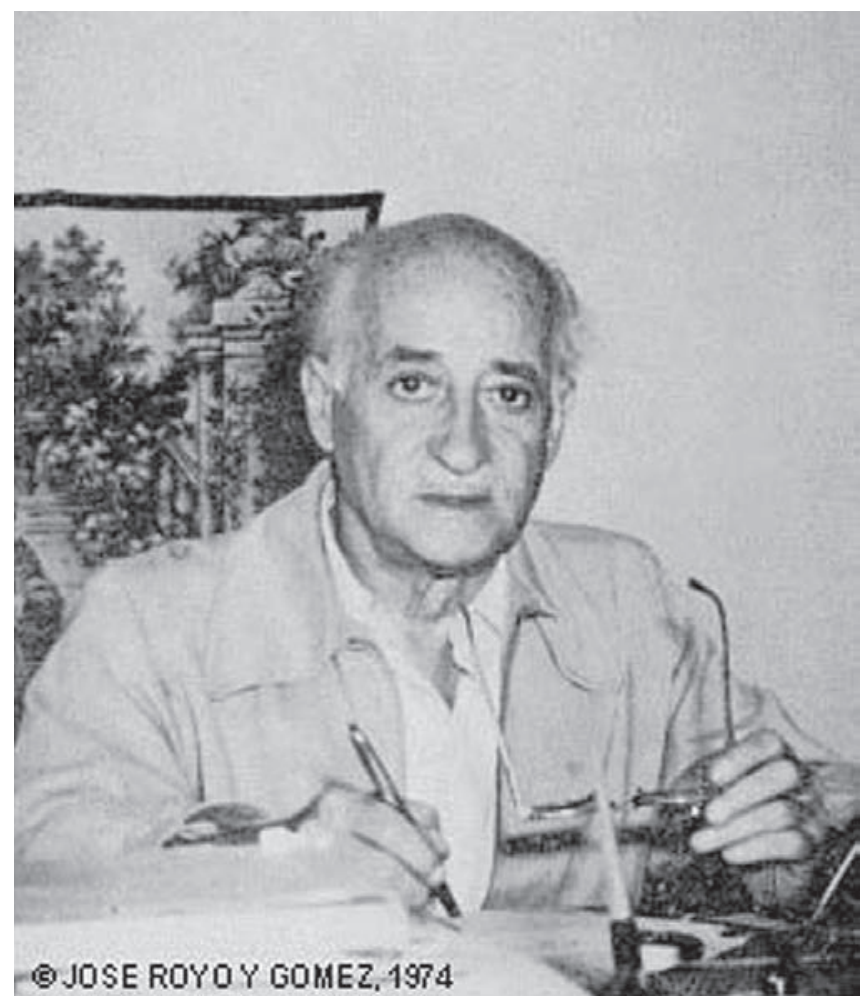

Figura 3. José Royo y Gómez (1895-1961). 
cente Sos Baynat (1895-1992), catedrático de Instituto y doctor en Ciencias Naturales y que fue discípulo de Royo y Gómez (Alcalá \& Alcalá, 1996).

La primera comunicación de Royo y Gómez referida a los vertebrados fósiles de Morella tuvo lugar en la sesión del 6 de marzo de 1918 de la Real Sociedad Española de Historia Natural. En ella mencionó la notificación realizada por el profesor Beltrán (1918) a la sección de Valencia de la propia Sociedad en la sesión del 31 de enero. En dicha notificación, Beltrán presentó varios restos de diversos reptiles del Cretácico inferior de Morella, entre ellos, molares y trozos de hueso de Iguanodon. Royo y Gómez (1918) hizo observar la importancia de este hallazgo, pues consideró que podría servir para clasificar otros restos de la misma localidad depositados en el Museo Nacional de Ciencias Naturales de Madrid (MNCN) por Vilanova y Piera o por él mismo, ya que en 1914, había encontrado una costilla de Iguanodon.

En 1920 estudió los yacimientos "weáldicos" del Maestrazgo y además de determinar varios invertebrados, señaló el hallazgo de nuevos materiales de dinosaurios, clasificándolos como "Iguanodon talla Mantelli" y concluyó que los yacimientos de vertebrados se producían por acarreos fluviales por lo cual se hacía difícil la localización de esqueletos completos (Royo y Gómez, 1920). En el Congreso de la Asociación Española para el Progreso de las Ciencias del año 1921, celebrado en Oporto, dentro de su trabajo sobre la facies continental en el Cretácico inferior ibérico, presentó una serie de restos, según él, probablemente de Megalosaurus (Royo y Gómez, 1921).

En los años 1922 y 1923 realizó un reconocimiento y una excavación cerca de la carretera de Morella a Cinctorres, entonces en construcción, en la cual, según Royo y Gómez (1926b), se localizaron varias vértebras caudales y una cabeza de fémur de un gran saurópodo. En la sesión del 2 de abril de 1924, presentó una nota sobre reptiles gigantescos del "Weáldico" de Morella y exhibió algunos materiales recolectados por los Padres Escolapios de la localidad (Royo y Gómez, 1924). De entre las numerosas fichas fotográficas que realizó, se encuentra una de un puente en la carretera anteriormente citada con una nota manuscrita que indica (Fig. 4):

"Puente de la carretera de Cinctorres en donde están enterrados los restos de vertebrados Weáldicos aparecidos en la trinchera del fondo" Marzo, 1924.

En la sesión del 6 de mayo de 1925, dio cuenta del envío de fósiles de Morella y Benageber, entre otros, del profesor Beltrán, para proceder a su estudio. Royo y Gómez destacó la presencia de restos de Megalosaurus y del saurópodo Cetiosaurus? (Royo y Gómez, 1925).

Entre las diversas publicaciones de 1926, el trabajo más extenso lleva por título "Los descubrimientos de reptiles gigantescos de Levante". Siendo un formato divulgativo,

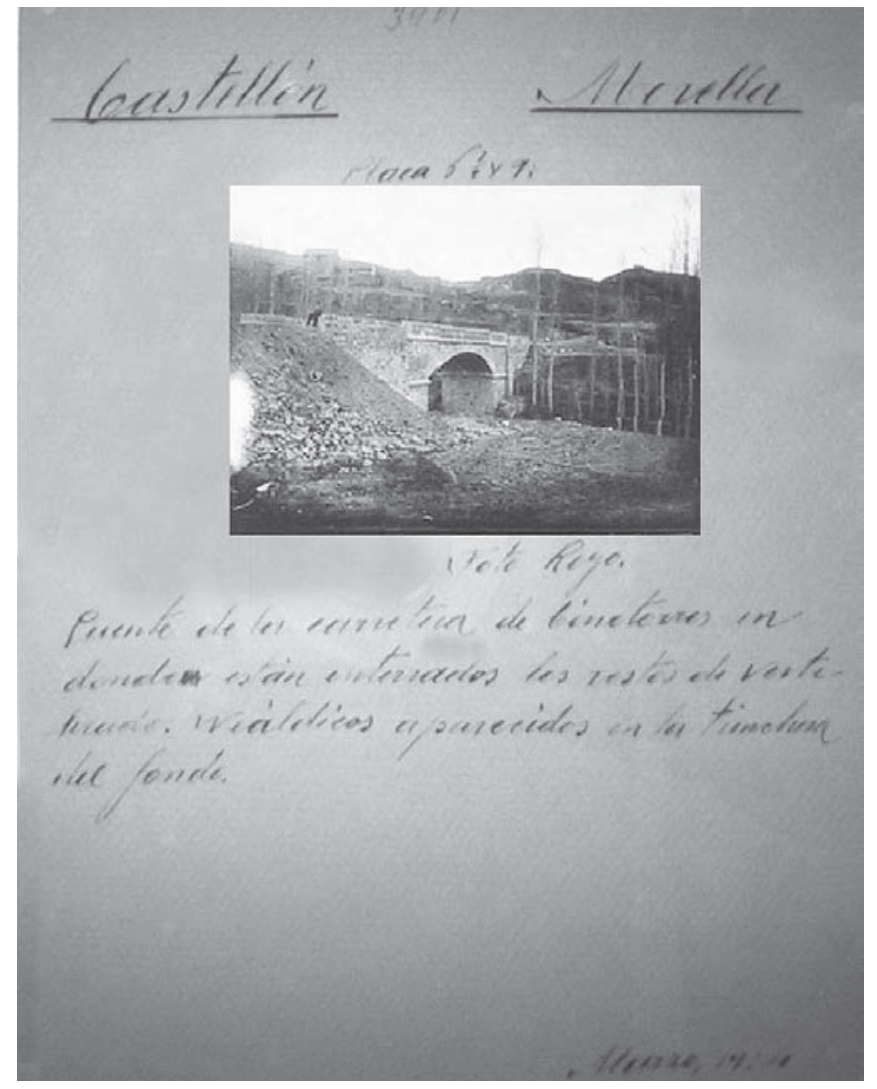

Figura 4. Fotografía original de Royo del puente de la carretera de Morella a Cinctorres. Archivo del MNCN de Madrid.

Royo's original photograph of the bridge of the highway from Morella to Cinctorres. Archivo del MNCN de Madrid.

es el único trabajo de Royo y Gómez que incluye figuras de dinosaurios. Identifica tres dinosaurios diferentes: un saurópodo (Cetiosaurus?), un ornitópodo (Trachodon?) y un terópodo (Megalosaurus). También se queja de la utilización de restos fósiles para la construcción del puente de la carretera de Morella a Cinctorres (Royo y Gómez, 1926a). En el mismo año con motivo del XIV Congreso de Geología Internacional de Madrid, realizó una exposición con restos de dinosaurios de Morella (Santafé \& Casanovas, 1993).

En 1927 presentó internacionalmente los dinosaurios españoles, determinando para Morella un conjunto de dinosaurios integrado por saurópodos, ornitópodos, terópodos y estegosáuridos (Royo y Gómez, 1927). Finalmente, en 1928 se publicó la comunicación que presentó dos años antes en el XIV Congreso de Geología Internacional de Madrid (Royo y Gómez, 1928).

Tanto Vilanova y Piera como Royo y Gómez depositaron los materiales de sus investigaciones en el MNCN. Según la documentación del Museo, Royo y Gómez aún continuó entregando restos de Morella hasta 1933 (Ruiz- 
Omeñaca \& Pereda-Suberbiola, 1999). Los restos fósiles de vertebrados, que se conservan en la actualidad en el MNCN, suponen un total de 1.309 piezas o fragmentos. La mayor parte de los fragmentos son de pequeño tamaño y muy difíciles de determinar. Dichos restos están catalogados en un total de 84 fichas de yacimientos de Morella y Vallibona, de las que 52 de Morella y 4 de Vallibona hacen referencia a restos de dinosaurios. Ruiz-Omeñaca $e t$ al. (2003) consideran que entre las piezas de Morella hay restos de terópodos (Theropoda indet., Tetanurae indet.), saurópodos (Sauropoda indet., Titanosauriformes indet., Brachiosauridae indet.), y ornitópodos (Ornithopoda indet., cf. Hypsilophodon sp., "Iguanodontidae" indet.).

\section{LA PRIMERA MONOGRAFÍA DE DINOSAURIOS}

Aunque hay referencias a restos aislados de Morella relacionados con Iguanodon (ver Lapparent, 1966), la investigación sobre dinosaurios en Morella quedó paralizada hasta prácticamente la década de los 70 . En ese momento, un aficionado local, Francisco Yeste López, interesado por el mundo de los fósiles y minerales, rastreó los afloramientos de la Formación Arcillas de Morella y localizó algunos yacimientos interesantes como El Beltrán (Tejería Azuvi) y Mas Romeu (Santafé, 1978; Santafé et al., 1979). Su labor puso en alerta al Ayuntamiento de Morella, quien solicitó la colaboración, a través de la Diputación de Barcelona, del Instituto de Paleontología Miquel Crusafont de Sabadell. De 1978 a 1980, el Instituto, bajo la dirección técnica de los doctores José Vicente Santafé y Lourdes Casanovas, realizó varias campañas de excavación en los yacimientos de Mas Romeu (Fig. 5), Mas Macià Querol y El Canteret aportando una gran cantidad de restos.

Los materiales fósiles recuperados permitieron la realización de varias comunicaciones (Santafé, 1978; Santafé \& Casanovas, 1979; Santafé et al., 1979) y en 1982 la primera monografía publicada en España sobre dinosaurios (Santafé et al., 1982a), además de un pequeño libro divulgativo (Santafé et al., 1982b). La monografía significó una primera definición geológica amplia de la Formación Arcillas de Morella (denominada entonces Capas rojas). También permitió establecer una serie de consideraciones tafonómicas y paleoecológicas, así como la determinación de un conjunto faunístico de dinosaurios compuesto por dos terópodos (Coelurosauridae indet. y Megalosauridae indet.), un saurópodo (Brachiosauridae indet.), un anquilosaurio (?Nodosauridae indet.) y un ornitópodo (Iguanodon bernissartensis) (Sanz et al., 1982).

En 1983 los mismos investigadores presentan una publicación sobre restos del dinosaurio ornitópodo Hypsilophodon. Se trataba de un conjunto de metatarsales y falanges localizados en el yacimiento de la Tejería Milián. Las

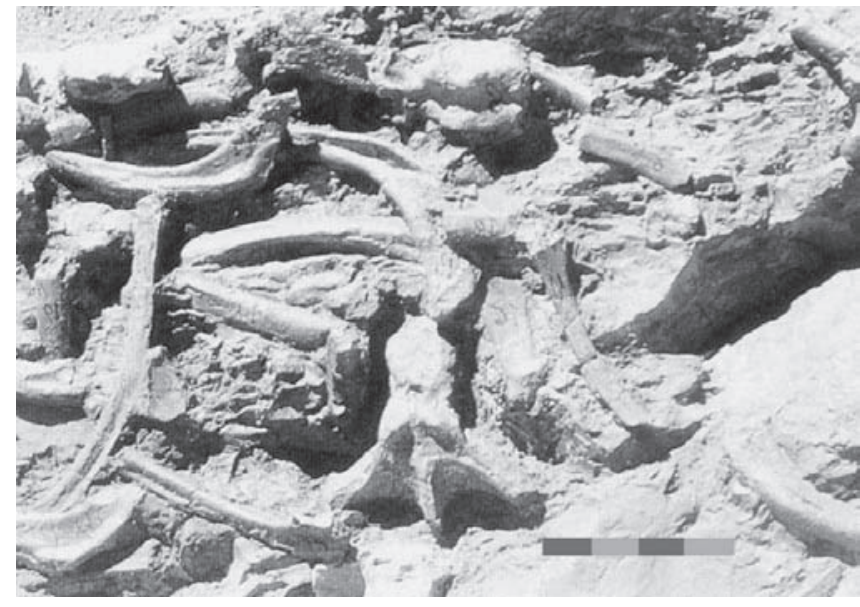

Figura 5. Depósito de concentración con restos de Iguanodon del yacimiento de Mas Romeu, Morella, Castellón (Cretácico Inferior). Escala $=20 \mathrm{~cm}$.

Concentration deposit of Iguanodon bones in Mas Romeu, Morella, Castellón (Lower Cretaceous). Scale $=20 \mathrm{~cm}$.

características de los restos permitieron a los autores proponer al hipsilofodóntido de Morella como Hypsilophodon sp., probablemente $H$. foxii (ver Sanz et al., 1983).

Aunque los trabajos científicos finalizaron en esa época, Francisco Yeste continuó con la labor de recuperación de restos, destacando de entre ellos, un sacro completo de Iguanodon cf. bernissartensis del yacimiento de la Tejería Azuvi y un húmero y un pubis de un saurópodo braquiosáurido del yacimiento de Mas de Eroles (Yagüe et al., 2001). Por otra parte, otro aficionado, Juan Cano Forner, de la población de San Mateo, también realizó labores extractivas en yacimientos de la comarca, incrementando su colección particular con restos muy interesantes de dinosaurios, especialmente del género Iguanodon, depositados hoy en su colección museográfica.

\section{EL MUSEO "TEMPS DE DINOSAURES"}

En 1994 por iniciativa del Ayuntamiento de Morella, bajo la coordinación del autor y la colaboración de José Vicente Santafé y Lourdes Casanovas, se creó el Museo monotemático "Temps de dinosaures. El Cretaci a Morella" (Fig. 6). Para ello, se utilizó la colección conservada en el propio Ayuntamiento correspondiente a las excavaciones del Instituto de Paleontología de Sabadell, así como otros restos en posesión de algunos particulares. El Museo está situado en las dos torres medievales que flanquean la puerta de San Miguel del cinturón amurallado de la ciudad. La concepción y distribución del Museo pretende destacar la singularidad de los huesos, con la voluntad, además, de ser un ejemplo de exposición educativa y divulgativa [e.g., 


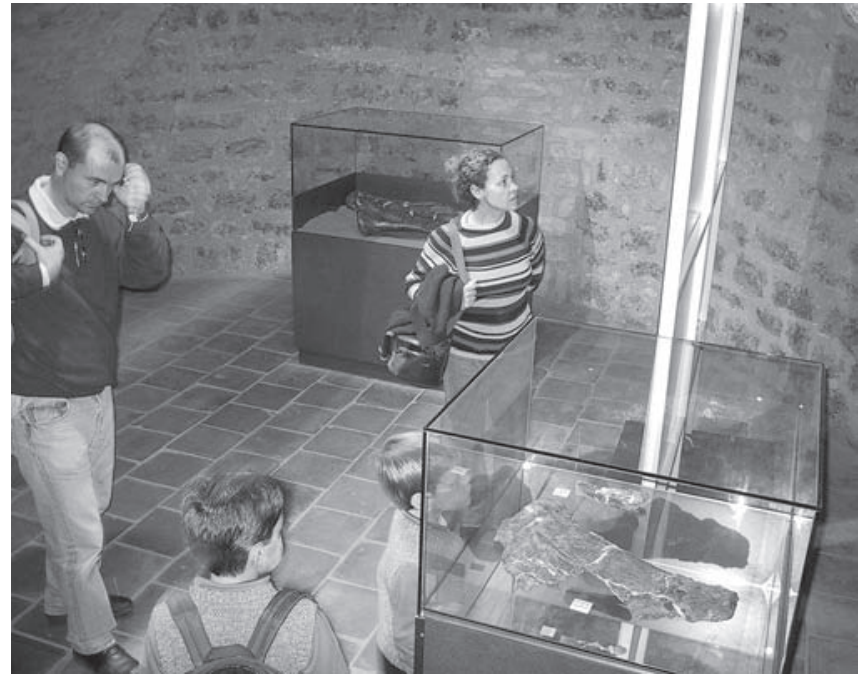

Figura 6. Sala con restos de saurópodo. Museo "Temps de dinosaures", Morella, Castellón.

Expositive space at the Museum "Temps de dinosaures”, Morella, Castellón with sauropods remains.

cada pieza o grupo de piezas expuestas tiene su propio panel explicativo, hay una guía del Museo para cada visitante o existe la posibilidad de adquirir varias publicaciones, entre ellas un cómic infantil del propio Museo (Gasulla \& Bayés, 1997)]. El recorrido museístico se realiza a través de las distintas salas de las torres, prestando una atención especial a los restos del dinosaurio más abundante y mejor conocido del registro fósil morellano como es Iguanodon. El número de visitantes se eleva a una media de más de 20.000 anuales, a pesar de las diversas dificultades que supone su visita, motivadas por las características de los edificios (arquitectura militar medieval).

También en el mismo año, se fundó la asociación "Amics de la Paleontologia de Morella" para respaldar los trabajos de investigación, vigilancia y conservación de los yacimientos y sus restos fósiles, además de promover y organizar varias jornadas anuales de estudios paleontológicos.

Una de las actuaciones conjuntas del Museo y de la asociación en colaboración con otras instituciones fue la realización de una exposición itinerante con una parte de las colecciones fósiles, visitando ciudades como Lisboa y Tomar en Portugal y en la provincia de Castellón, la propia ciudad de Castellón, Onda, Almassora o Les Coves de Vinromà. Además en el año 2001, por primera vez, el Museo Nacional de Ciencias Naturales de Madrid acogió una exposición de dinosaurios de Morella en la que se unieron parte de las colecciones de ambos museos. Los fósiles aportados por el MNCN correspondieron a parte de la colección depositada en su momento por Royo y Gómez.

\section{LA INVESTIGACIÓN ACTUAL}

En 1997, de nuevo se incrementó el registro fósil con el descubrimiento del yacimiento de icnitas de Vallivana (Fig. 7), primeras evidencias indirectas de la presencia de dinosaurios en Morella. Dicho descubrimiento fue bastante curioso, ya que el niño que localizó el yacimiento se encontraba, precisamente, jugando en la búsqueda de huellas fósiles de dinosaurios. El yacimiento está incluido dentro de una potente serie de calizas lacustres neocomienses ricas en restos de carofitas. El conjunto de huellas se encuentra sobre la superficie de una placa calcárea gris, con un pronunciado buzamiento de dirección sudeste. Contiene un rastro compuesto por cuatro icnitas y varias icnitas aisladas, todas tridáctilas que han sido atribuidas a dinosaurios terópodos. En el 2003, se procedió a ampliar la zona de estudio del yacimiento. La nueva zona descubierta presenta una alta densidad icnológica y, en muchos de los casos, las icnitas se encuentran incompletas debido a una intensa superposición que hace difícil la delimitación de los contornos y la identificación de las mismas. Se trata

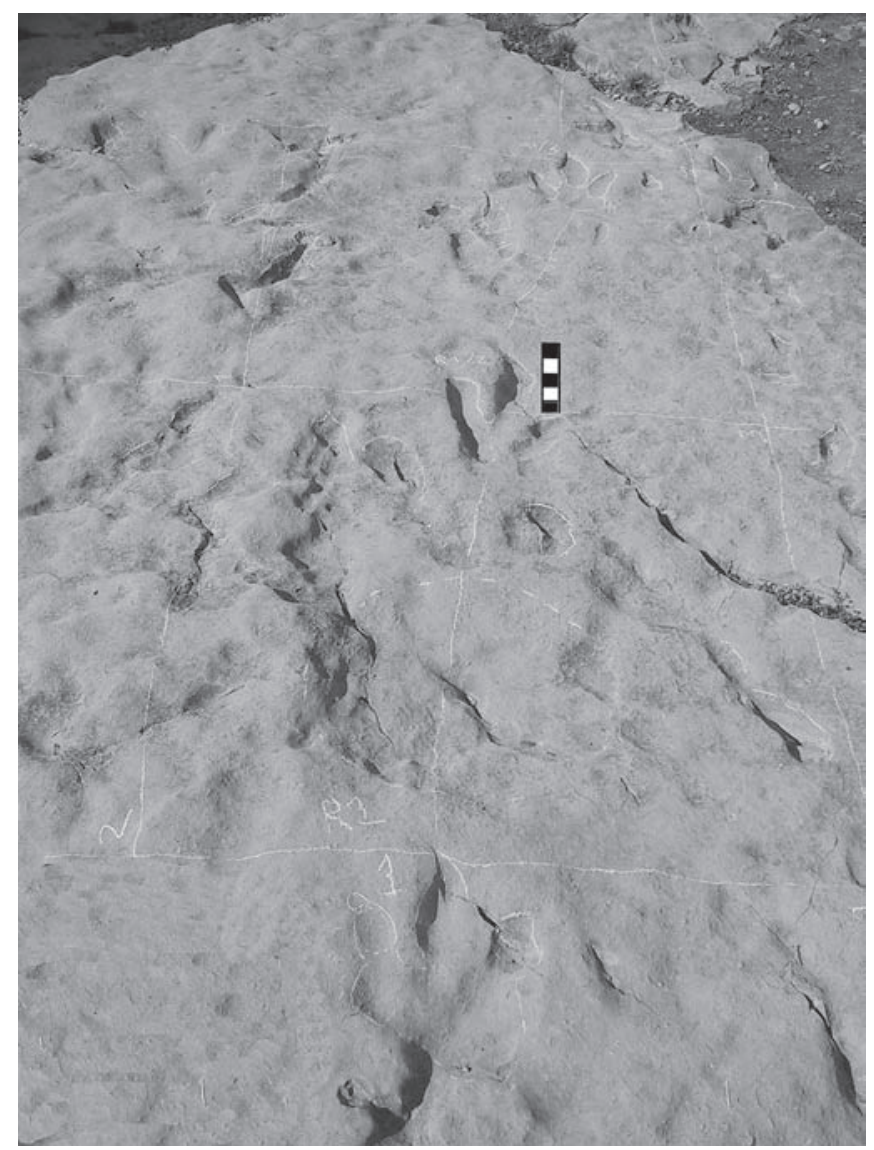

Figura 7. Yacimiento de icnitas de terópodos de Vallivana, Morella, Castellón (Cretácico Inferior). Escala = $20 \mathrm{~cm}$.

Theropods tracks in Vallivana, Morella, Castellón (Lower Cretaceous). Scale $=20 \mathrm{~cm}$. 
de un claro ejemplo de dinoturbación de intensidad media (Moratalla et al., 2003).

En 1998 se descubrió un gran fragmento de húmero derecho de iguanodóntido en la zona del Mas de Romeu, uno de los yacimientos bien conocidos de Morella. El material fue depositado en el Centre de Natura "El Termet" de Vila-real. Fue asignado a Iguanodon sp. (Ruiz-Omeñaca \& Santos-Cubedo, 1998).

Con la apertura de la cantera de arcillas del Mas de la Parreta, en el año 2000, la investigación paleontológica ha tomado un nuevo impulso. La realización de un control paleontológico permanente, con la contratación de un técnico, José Miguel Gasulla, por parte de la empresa Vega del Moll, S.A., adjudicataria de la explotación, ha dado sus frutos con la localización de siete yacimientos diferentes dentro de la propia cantera y la recuperación de un elevado número de restos (Fig. 8). Además ha permitido reunir a diversos especialistas con el fin de concretar el conocimiento de la Formación Arcillas de Morella y su registro fósil. Así, José Luís Sanz y Francisco Ortega de la Universidad Autónoma de Madrid, colaboran en la recuperación de los restos esqueléticos y su clasificación. Un equipo de investigadores encabezado por Ramón Salas, de la Universidad de Barcelona, se ocupa de los aspectos sedimentológicos y estratigráficos de los yacimientos (Gàmez, 2002; Salas, 2003; Gàmez et al., 2003; Salas et al., 2003). Carles Martín-Closas y Xavier Delclòs, también

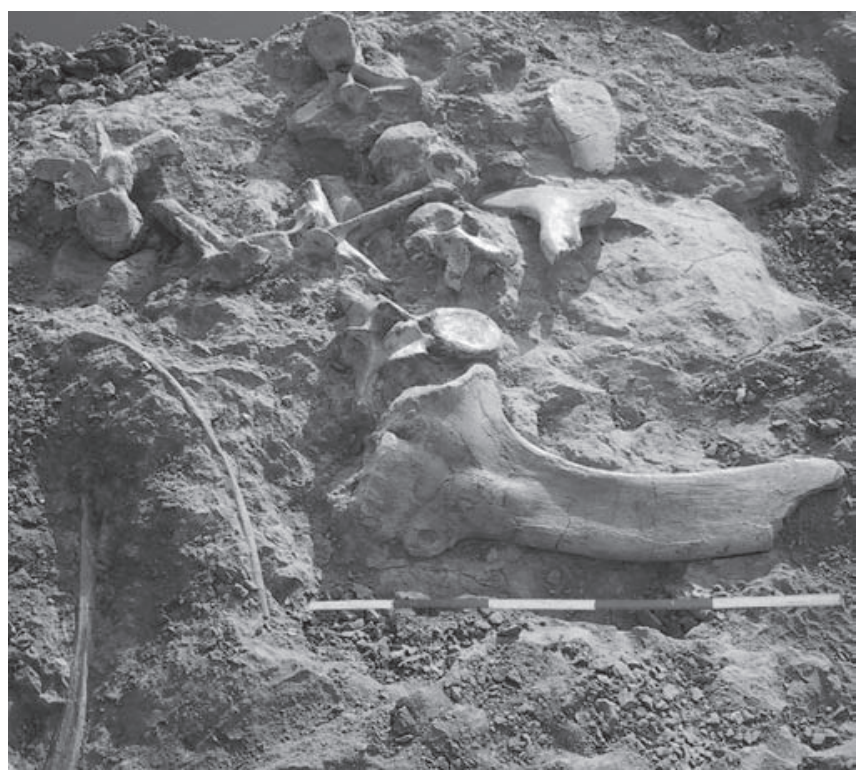

Figura 8. Depósito de concentración con restos de Iguanodon del yacimiento CMP-5 de la Cantera del Mas de la Parreta, Morella, Castellón (Cretácico inferior). Escala $=100 \mathrm{~cm}$.

Concentration deposit with bones of Iguanodon from the site CMP-5 in the Cantera Mas de la Parreta, Morella, Castellón (Lower Cretaceous). Scale = $100 \mathrm{~cm}$. de la Universidad de Barcelona, realizan estudios paleobotánicos y tafonómicos (Martin-Closas \& Gómez, 2003; Salas et al., 2003).

Desde el año 2001 se están revisando algunos taxones de dinosaurios de los yacimientos de Morella. Ruiz-Omeñaca (2001) considera que los restos del ornitópodo Hypsilophodon sp., estudiados por Sanz et al. (1983), no son diagnósticos y los determina como Hypsilophodontidae indet. Además asigna a cf. Hypsilophodon sp. una pieza dental de la Colección Royo y Gómez del MNCN, cuyo origen probablemente fuera de algún afloramiento de la Formación Arcillas de Morella. Yagüe et al. (2001) revisan los restos de saurópodos de los yacimientos de Morella estudiados en la monografía de 1982. Dicha revisión también incluye los nuevos restos de saurópodo del yacimiento de Mas de Eroles, citados con anterioridad. Las conclusiones apuntan a la identificación de una nueva especie de saurópodo dentro del grupo de los braquiosáuridos.

En el 2002 se realizó un estudio en una vértebra dorsal media de Iguanodon perteneciente al yacimiento CMP-5 de la cantera del Mas de la Parreta (Fig. 9). Se aplicó un diagnóstico diferencial a una patología que sufría dicha vértebra en la cápsula articular de su articulación vertebrocostal izquierda. Las conclusiones indican que la alteración

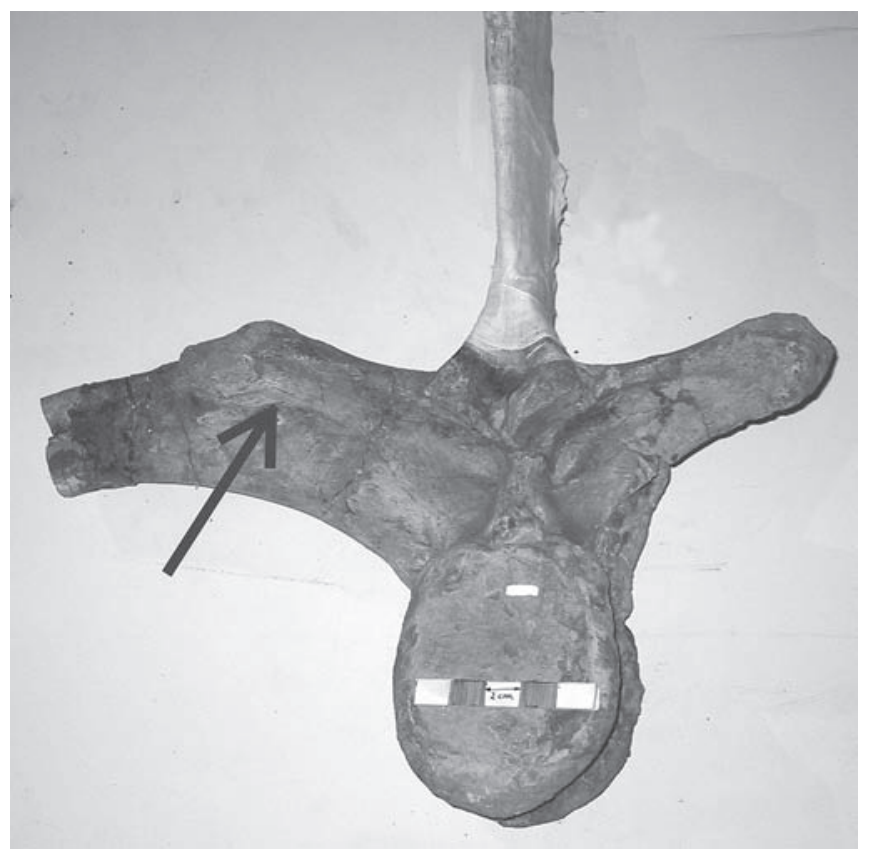

Figura 9. Vértebra dorsal media de Iguanodon del yacimiento CMP-5 de la Cantera del Mas de la Parreta, Morella, Castellón (Cretácico inferior). La flecha indica la deformación patológica. Escala $=10 \mathrm{~cm}$.

Middle dorsal vertebra of Iguanodon (site CMP-5 in the Cantera Mas de la Parreta, Morella, Castellón (Lower Cretaceous)). The arrow shows the pathological deformation. Scale $=10 \mathrm{~cm}$. 


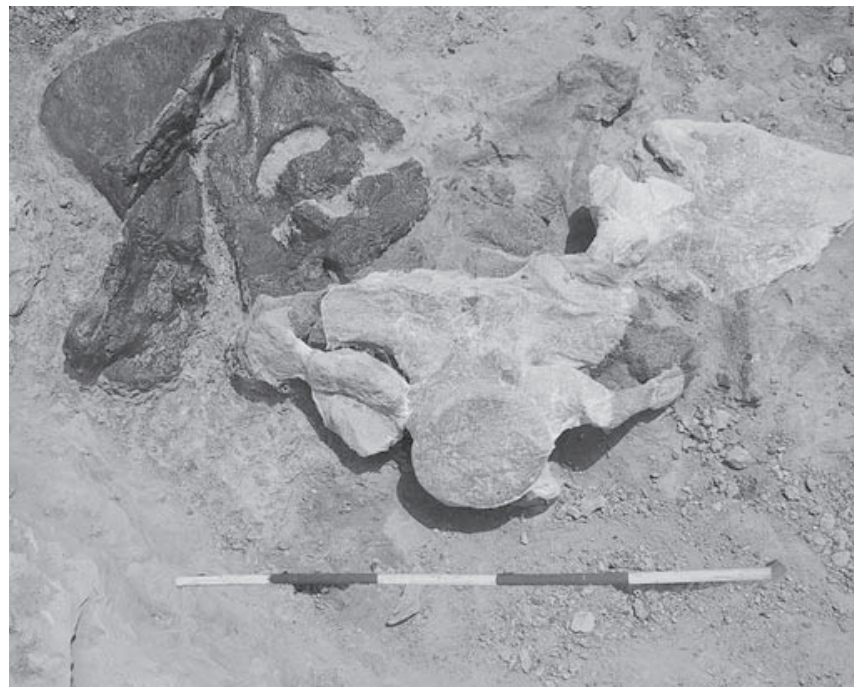

Figura 10. Un sacro y dos iliones de saurópodo del yacimiento CMP-3 de la Cantera del Mas de la Parreta, Morella, Castellón (Cretácico inferior). Escala $=100 \mathrm{~cm}$. Sacrum and the two ilia of a sauropod in situ (site CMP-3 in the Cantera Mas de la Parreta, Morella, Castellón (Lower Cretaceous). Scale $=100 \mathrm{~cm}$.

se pudo producir por un proceso traumático o congénito (Cambra-Moo et al., 2002).

Los depósitos del yacimiento CMP-3 de la cantera del Mas de la Parreta han proporcionado una amplia y diversa muestra de fósiles de vertebrados constituida no solo por varios tipos de dinosaurios sino también por tiburones, peces óseos, tortugas, cocodrilos y plesiosaurios (Fig. 10). Estos últimos son de cierta relevancia ya que los restos de estos reptiles marinos son escasos en el Cretácico inferior europeo y particularmente en el ibérico (Yagüe et al., 2002). En cuanto a restos de dinosaurios aparecen representados varios taxones (Theropoda, Sauropoda, Ornithopoda y Thyreophora). De este último se han identificado varios elementos de la armadura dérmica de Polacanthus sp. (Gasulla et al., 2003). Canudo et al. (2004) consideran que los restos del anquilosaurio Nodosauridae? indet., estudiados también en la monografía de 1982, corresponden a Ankylosauria indet.

\section{CONCLUSIONES}

Probablemente no hay muchos lugares en España en los que la investigación de dinosaurios en particular y la paleontológica en general tenga tan larga tradición como en Morella. Desde el último tercio del siglo XIX dicha investigación, no exenta de altibajos, ha ido incrementado y definiendo el conocimiento del conjunto faunístico de dinosaurios de los yacimientos de Morella. El incremento de la investigación actual ha sido muy destacado, como lo demuestra el hecho de que veinte de las referencias utilizadas en el presente trabajo se han realizado en los últimos diez años. Sin duda, una parte importante de este incremento ha sido motivada por la elección de Morella como sede de las XIX Jornadas de la Sociedad Española de Paleontología (SEP), ya que en estas Jornadas se han presentado varios trabajos de investigación sobre los yacimientos morellanos.

Por otra parte y no menos importante, poco a poco, se está consolidando el aspecto divulgativo de los recursos paleontológicos con la puesta en valor de dichos recursos, tanto por las entidades públicas (Museo de Morella, Asociación "Amics de la Paleontologia" o la propia SEP) como por la iniciativa privada (Vega del Moll, S.A.).

Por último, el nivel de conocimiento actual de los diversos dinosaurios de Morella nos permite componer un conjunto taxonómico intergrado por: terópodos (Theropoda indet., Tetanurae indet., Megalosauridae indet., Coelurosauridae indet.), saurópodos (Sauropoda indet., Titanosauriformes indet., Brachiosauridae indet.), ornitópodos (Hypsilofodontidae indet., cf. Hypsilophodon sp., "Iguanodontidae" indet., Iguanodon sp., I. cf. bernissartensis, I. bernissartensis) y tireóforos (Ankylosauria indet., Polacanthus sp.).

\section{AGRADECIMIENTOS}

Quiero expresar mi agradecimiento a Patxi Ortega de la Unidad de Paleontología de la Universidad Autónoma de Madrid, por su perpetua colaboración y sus sugerencias. Así mismo quiero agradecer la colaboración prestada por Carlos Martinez y Ana García del departamento de Geología de la Universidad de Valencia para conocer mejor la figura de Nicolás Ferrer y Julve. A Joaquin Moratalla, del IGME, por sus enseñanzas en el campo de la Icnología. A Manuel Domingo García Sanz por su actitud siempre entusiasta a pesar de las dificultades.

Un agradecimiento especial a la Sociedad Española de Paleontología por decidir que Morella, mi ciudad, fuera la sede de las XIX Jornadas de la SEP. También a los compañeros y amigos del comité organizador, a las instituciones y colaboradores y a todos los participantes en las jornadas. Seguro que mi agradecimiento es el de todos los/las morellanos/as.

Fotografías: fig. 5 realizada por Joan Salangera; figs. 6 a 10 realizada por José Miguel Gasulla.

\section{BIBLIOGRAFÍA}

Alcalá, B. \& Alcalá, L. 1996. La paleontología castellonense explorada por José Royo Gómez. Geogaceta, 19, 177-180. 
Beltrán, F. 1918. Sesión del 6 de marzo de 1918. Secciones del Boletín Real Sociedad Española de Historia Natural, $18,134$.

Cambra-Moo, O., Ortega, F., Gasulla, J.M. \& Yagüe, P. 2002. "Diga 33". Aplicación del diagnóstico diferencial a una patología vertebral en Iguanodon (Cretácico inferior. Morella, Castellón). Libro de Resúmenes del Congreso Internacional sobre Dinosaurios y otros reptiles mesozoicos de España. Logroño, 9.

Canudo, J.I., Ruiz-Omeñaca, J.I. \& Cuenca-Bescós, G. 2004. Los primeros dientes de anquilosaurio (Ornithischia: Thyreophora) descritos en el Cretácico inferior de España. Revista Española de Paleontología, 19, 33-46.

Ferrer y Julve, N. 1877. España. Antiguo Reino de Valencia. Morella. Colección de Fósiles recogida y presentada por el $D^{r}$. D. Nicolás Ferrer y Julve. Arxiu Universitat València, documento $\mathrm{c} / 306 / 5$.

Gàmez, D. 2002. Estratigrafía y sedimentología de la Formación Arcillas de Morella del Aptiense inferior (Provincia de Castellón). Tesis de Licenciatura. Departament d'Estratigrafia, Paleontologia i Geociències Marines. Universitat de Barcelona, 150 pp. (inédita).

Gàmez, D, Panciotti, P., Colombo, F \& Salas, R. 2003. La Formación Arcillas de Morella (Aptiense inferior), Cadena Ibérica Oriental: Caracterización sedimentológica. Libro de resúmenes de las XIX Jornadas de la Sociedad Española de Paleontología. Morella, 78.

García, A. 1999. El Museo del Departamento de Geología. En: Los Tesoros de la Universitat de València. Publicacions de la Universitat de València, Patronat Cinc Segles, 271-282.

Gasulla, J.M. \& Bayés, P. 1997. Petita història del Museu de Morella. Temps de dinosaures. Col-lecció Petites històries, 92, Ed. Mediterrània, Barcelona, 16 pp.

Gasulla, J.M, Ortega, F., Pereda-Suberbiola, X. \& Sanz, J.L. 2003. Elementos de la armadura dérmica del dinosaurio anquilosaurio Polacanthus (Cretácico inferior, More1la, Castellón, España). Libro de resúmenes de las XIX Jornadas de la Sociedad Española de Paleontología. Morella, 83.

Gozalo, R. \& Salavert, V. 1995. Joan Vilanova i Piera. València, 1821-Madrid, 1883. Geòleg, paleontòleg i prehistoriador. En: Ciència i Tècnica als Països Catalans: una aproximació biogràfica (dirs. J.M. Camarasa \& A. Roca Rosell). Fundació Catalana per a la Recerca, 1, Barcelona, 289-313.

Lapparent, A. F. 1966. Nouveaux gisements de Reptiles Mesozoïques en Espagne. Notas y Comunicaciones del Instituto Geológico y Minero de España, 84, 103-110.

Martín-Closas, C. \& Gómez, B. 2003. El género Frenelopsis en el Cretácico inferior ibérico. Libro de resúmenes de las XIX Jornadas de la Sociedad Española de Paleontología. Morella, 20-21.

Moratalla, J.J., Gasulla, J.M., Salas, R. \& Martín-Closas, C. 2003. El yacimiento neocomiense (Cretácico inferior) de icnitas de dinosaurios terópodos de Vallivana (Morella, Castellón, España). Libro de resúmenes de las XIX Jornadas de la Sociedad Española de Paleontología. Morella, 121.
Orti, F. 1958. Historia de Morella. Tomo primero Prehistoria y Protohistoria. Ediciones Ortí, Benimodo, 136 pp.

Royo y Gómez, J. 1918. Sesión del 6 de marzo de 1918. Notas y comunicaciones del Boletín Real Sociedad Española de Historia Natural, 18, 9 y 133.

Royo y Gómez, J. 1920. Los yacimientos weáldicos del Maestrazgo. Boletín Real Sociedad Española de Historia Natural, 20, 261-267.

Royo y Gómez, J. 1921. La facies continental en el Cretácico inferior ibérico. Asociación Española para el Progreso de las Ciencias. Congreso Oporto, 6, 221-236.

Royo y Gómez, J. 1924. Nota sobre reptiles gigantescos del Weáldico de Morella. Boletín Real Sociedad Española de Historia Natural, 24, 177.

Royo y Gómez, J. 1925. Sesión del 6 de mayo de 1925. Notas y comunicaciones del Boletín Real Sociedad Española de Historia Natural, 25, 251-252.

Royo y Gómez, J. 1926a. Los descubrimientos de reptiles gigantescos en Levante. Boletín de la Sociedad Castellonense de Cultura, 7, 147-162.

Royo y Gómez, J. 1926b. Los vertebrados del Cretácico Español de Facies Weáldica. Boletín del Instituto Geológico de España, 47, 171-176.

Royo y Gómez, J., 1927. Sur le faciés wealdien d'Espagne. Compte Rendu sommaire des Séances de la Societé géologique de France, 11, 125-128.

Royo y Gómez, J. 1928. Les vertébrés du wealdien espagnol. Compte Rendu XIV Session du Congrès Géologique International, Madrid, 1926, 4, 2039-2042.

Ruiz-Omeñaca, J.I. 2001. Dinosaurios hipsilofodóntidos (Ornithischia: Ornithopoda) en la Península Ibérica. Actas I Jornadas Internacionales sobre Paleontología de Dinosaurios y su Entorno. Salas de los Infantes, 175-266.

Ruiz-Omeñaca, J.I. \& Pereda Suberbiola, X. 1999. Un documento inédito de Royo y Gómez sobre los dinosaurios del Levante. Temas Geológico-Mineros, 26, 111-112.

Ruiz-Omeñaca, J.I. \& Santos-Cubedo, A. 1998. Un húmero de iguanodóntido (Ornithischia: Ornithopoda) del Cretácico inferior (Aptiense) de Morella (Castellón, España). Geogaceta, 24, 279-282.

Ruiz-Omeñaca, J.I, Pereda Suberbiola, X. \& Company, J. 2003. Juan Vilanova, José Royo y Gómez, y los dinosaurios de Morella en las colecciones del Museo Nacional de Ciencias Naturales de Madrid. Libro de resúmenes de las XIX Jornadas de la Sociedad Española de Paleontología. Morella, 150-151.

Salas, R. 2003. Paleogeografía de la Cadena Ibérica Oriental durante el Cretácico inferior. Libro de resúmenes de las XIX Jornadas de la Sociedad Española de Paleontología. Morella, 22-23.

Salas, R., Colombo, F., Gàmez, D., Gómez, B., Gasulla, J.M., Martín-Closas, C., Moratalla, J., Panciotti, P., Querol, X. \& Solé de Porta, N. 2003. Guía de la Excursión. XIX Jornadas de la Sociedad Española de Paleontología. Morella, $31 \mathrm{pp}$.

Santafé J. 1978. Campanyes de Morella. Butlletí Informació del Institut de Paleontologia de Sabadell. 10, 26-27.

Santafé, J.V. \& Casanovas, M.L. 1979. Situación estratigráfica 
de los yacimientos de dinosaurios del Levante Español. Butlletí Informació del Institut de Paleontologia de Sabadell, 11, 29-33.

Santafé, J.V. \& Casanovas, M.L. 1993. Dinosaurios en la Comunidad Valenciana. Generalitat Valenciana, 205 pp.

Santafé, J.V., Casanovas, M.L., Sanz, J.L. \& Calzada, S. 1979. Los Dinosaurios de Morella (Nota preliminar). Acta geológica hispánica, 5, 149-154.

Santafé, J.V., Casanovas, M.L., Sanz, J.L. \& Calzada, S. 1982a. Geología y Paleontología (Dinosaurios) de las Capas rojas de Morella (Castellón, España). Diputación Provincial de Castellón y Diputación de Barcelona, 169 pp.

Santafé, J.V., Casanovas, M.L., Sanz, J.L. \& Calzada, S. 1982b. Morella y su fauna fósil. Diputación de Castellón, $59 \mathrm{pp}$.

Sanz, J.L. 1996. José Royo y Gómez y los dinosaurios españoles. Geogaceta, 19, 167-168.

Sanz, J.L., Casanovas, M. L. \& Santafé, J. V. 1982. Paleontología. In: Geología y Paleontología (Dinosaurios) de las Capas rojas de Morella (Castellón, España) (J. V. Santafé, M. L. Casanovas, J. L. Sanz \& S. Calzada). Diputación Provincial de Castellón y Diputación de Barcelona, 69-169.

Sanz, J.L., Santafé, J.V. \& Casanovas, L. 1983. Wealden
Ornithopod dinosaur Hypsilophodon from the Capas rojas Formation (Lower Aptian, lower Cretaceous) of Morella, Castellón, Spain. Journal of Vertebrate Paleontology, 3, 39-42.

Segura y Barreda, J. 1868. Morella y sus aldeas. Tomo 2. Imp. de F. Javier Soto, Morella, 490 pp.

Vilanova y Piera, J. 1872. Compendio de Geología. Imprenta de Alejandro Gomez Fuentenebro, Madrid, 588 pp.

Vilanova y Piera, J. 1873. Sesión del 5 de febrero de 1873. Acta de la Sociedad Española de Historia Natural, 2, 8.

Yagüe, P., Upchurch, P., Sanz, J.L. \& Gasulla, J.M. 2001. New Sauropod material from the Early Cretaceous of Spain. $49^{\text {th }}$ Annual Symposium of Vertebrate Paleontology and Comparative Anatomy. York (UK). 3-5/9/2001. www. svpca.org

Yagüe, P., Ortega, F., Noé, L.F., Gasulla, J.M. \& García, M.D. 2002. Reptiles marinos (Plesiosauria) del Aptiense inferior de Morella (Castellón). Libro de Resúmenes del Congreso Internacional sobre Dinosaurios y otros reptiles mesozoicos de España. Logroño, 71.

Manuscrito recibido: 1 de Diciembre, 2003 Manuscrito aceptado: 13 de Abril, 2005 